\title{
ERNEST COUMET ET L'HISTOIRE DE L'HISTOIRE DES SCIENCES
}

\author{
Pietro REDONDI
}

Je voudrais apporter mon témoignage sur un aspect qui a été au cœur de la recherche et de l'enseignement d'Ernest Coumet tout au long des séminaires qu'il a donnés au Centre Alexandre-Koyré en tant que directeur d'études de l'École des hautes études en sciences sociales.

Je pense à sa façon de mener de front l'enseignement et l'analyse de l'histoire des sciences en tant qu'objet historique. Coumet a en effet montré que les catégories, les périodisations spécifiques, les représentations du passé scientifique sont de plein titre l'effet d'une histoire, et d'une histoire qui reste à faire. De ce point de vue, et par comparaison avec l'enseignement de nos universités d'origine ou des séminaires classiques du Centre Alexandre-Koyré de la rue Colbert, l'enseignement de Coumet aura représenté pour deux générations de chercheurs une impulsion critique sans équivalent ailleurs.

L'impression que j'ai gardée du tout début de ses séminaires est d'ailleurs celle d'un certain exotisme par rapport à la géographie institutionnelle parisienne de l'histoire des sciences. Tout d'abord, lorsque, en 1980, Coumet commença son séminaire, les réunions se tenaient loin de tout lieu universitaire consacré : rue de la Tour, une annexe de l'École des hautes études où, dans des salles mal chauffées, se donnait rendez-vous un auditoire emmitoufflé de quelque trois ou quatre étudiants étrangers aiguillés vers ce lieu par le service d'orientation des thèses.

Parmi eux, se trouvait par exemple Emilios Metaxopoulos, qui après des séjours d'étude à Florence et à Londres préparait sous la direction d'Olivier Bloch une thèse sur Hobbes et avait la surprise de découvrir en Coumet un connaisseur aussi bien de la pensée post-néopositiviste de John Watkins que de l'histoire des idées de Paolo Rossi.

Même physiquement, avec sa figure ronde et sa nuque dégagée, Coumet apparaissait comme l'exact contraire de l'intellectuel parisien type. L' « exotisme» de ses séminaires tenait au profil singulier d'un historien des sciences venu à l'étude de l'art combinatoire de Marin Mersenne à traRevue de synthèse : $4^{\mathrm{e}}$ sér., ${ }^{\text {os }}$ 2-3-4, avr.-déc. 2001, p. 291-296. 
vers la logique mathématique de Lewis Carroll, l'empirisme de Rudolf Carnap et la théorie de la réfutabilité poppérienne: un érudit qui s'y connaissait en philosophie du langage et en théorie des jeux, bref, un historien analytique.

En témoignait son style pédagogique, dès qu'il prenait la parole, une fois ouverts sur la table ses grands carnets de notes parsemés de signets, et qu'il abordait l'analyse d'une œuvre et d'un auteur, cherchant toujours à discerner et à distinguer nettement dans leurs échafaudages conceptuels les présupposés et les options par rapport aux résultats d'interprétation auxquels elles conduisaient.

C'était le début des années quatre-vingt, et l'enseignement de l' « histoire des sciences » était alors dispensé à Paris dans deux institutions antagonistes. L'une, rue du Four, était l'Institut d'histoire des sciences et des techniques de la Sorbonne, fondé en 1932 par Abel Rey et qui avait vu se succéder comme directeurs Gaston Bachelard et Georges Canguilhem. Et l'autre, rue Colbert, était le Centre Alexandre-Koyré de l'École des hautes études en sciences sociales que Fernand Braudel et Alexandre Koyré avaient créé en 1958 auprès du Centre international de synthèse d'Henri Berr, le patriarche des Annales.

Rue du Four et rue Colbert : aux yeux des chercheurs de ma génération, ces lieux évoquaient deux façons irréductibles de faire de l'histoire des sciences : «philosophes » contre « scientifiques ${ }^{1}$. D'un côté, Suzanne Bachelard professait une histoire des sciences épistémologiquement orientée, ce qui signifiait une approche récurrente du passé scientifique d'après les valeurs et les connaissances actuelles, suivant les critères de l'épistémologie rationaliste de l'auteur de La Philosophie du non (1940). De l'autre côté, rue Colbert, se tenaient les séminaires de René Taton, élève lui aussi de Bachelard, ainsi que ceux de Pierre Costabel : il s'agissait d'une histoire des sciences de genre technique, factuel et biographique. Cela revenait à un métier d'érudition axé sur l'édition de textes et de correspondances des grands auteurs des sciences mathématiques classiques, d'après un idéal de reconstitution du passé que l'on faisait remonter à Paul Tannery et à Alexandre Koyré lui-même.

Tannery, Bachelard, Koyré. Derrière ces noms symboliques de promoteurs de la discipline se jouait une scission imaginaire entre philosophie et

1. J'ai essayé de reconstruire les antécédents de cette « cohabitation au sommet » de l'histoire des sciences parisienne dans l'article «French journals of the history of science. The checking of a deficit », in Journals and history of science, éd. Marco BerETTA, Claudio Pogliano et Pietro Redond, Florence, Olschki, 1998, p. 167-187. Voir, également, Enrico Castelli Gattinara, Les Inquiétudes de la raison, Paris, Vrin, 1998. Sur Berr et le milieu de la rue Colbert, voir les contributions au colloque Henri Berr et la culture du Xx siècle, dir. Agnès Biard, Dominique Bourel et Éric Brian, Paris, Albin Michel, 1997. Sur Georges Canguilhem, voir celles présentées au colloque Georges Canguilhem. Philosophe, historien des sciences, Paris, Albin Michel, 1993. 
science qui finissait par aboutir, de part et d'autre, à un retranchement sourd à toute discussion et confrontation de fond. Une drôle de guerre, mais qui faisait courir le risque de dessécher la richesse du débat dont avait bénéficié en France l'histoire des sciences jusqu'aux années soixante, c'est-à-dire l'époque de Georges Canguilhem, de Michel Foucault et de Louis Althusser.

Un risque de tarissement et d'isolement aussi, à l'image du retard avec lequel parut l'édition française du livre de Thomas Kuhn, La Structure des révolutions scientifiques, chez Flammarion seulement en 1977, alors que partout ailleurs ce livre, publié initialement en 1962, était depuis une décennie à la une de l'épistémologie historique.

Les années soixante avaient été profondément marquées par une discussion sur l'entreprise scientifique et son histoire, culminant au colloque entre kuhniens et poppériens organisé à Londres en 1965 à l'initiative de la British Society for Philosophy of Science et de la London School of Economics et dont la parution des actes en 1970 avait fait grand bruit ${ }^{2}$. Face à la crise du néopositivisme logique, quel était l'avenir pour l'histoire des sciences? Pouvait-on toujours parler d'une méthode scientifique souveraine ou devait-on accepter l'idée d'anarchisme méthodologique? La distinction entre une approche externaliste et une approche internaliste de l'histoire des sciences devait-elle être considérée comme définitive ou provisoire? Le colloque, intitulé Reason, experiment and mysticism in the Scientific Revolution, tenu à Capri en $1973^{3}$, témoignait bien des répercussions que ces remises en question épistémologiques avaient entraînées aussi sur le milieu restreint des spécialistes de la discipline.

En France, la perception du statut erratique d'une histoire des sciences en mutation rapide (« dont le désordre figure l'état d'un problème $\left.{ }^{4} »\right)$ est attesté par le numéro spécial que les Annales, en 1975, avaient consacré à «Histoire et sciences » et dont l'article phare était l'étude fameuse de Kuhn sur "Tradition mathématique et tradition expérimentale dans le développement de la physique ». Y était aussi publié un article de Coumet sur les systèmes de numération, à côté de textes de Claire Salomon-Bayet, Joseph Needham, Michel Serres, Charles Morazé, Mirko D. Grmek.

L'année précédente, dans la deuxième partie consacrée aux « Nouvelles approches» de la série programmatique Faire de l'histoire dirigée par

2. Paul Feyerabend, Thomas Kuhn, Imre Lakatos, Margaret Mastermann, Karl Popper, Stephan Toulmin, John Watkins, L. Pearce Williams, Criticism and growth of knowledge, Cambridge, Cambridge University Press, 1970, $1974^{3}$.

3. Reason, experiment and mysticism in the Scientific Revolution, éd. Maria Luisa RighinIBonelli et William Shea, New York, Science History Publ., 1975. Sur le tournant des années soixante, je me permets de renvoyer à mon article « The Scientific Revolution of the $17^{\text {th }}$ century. New perspectives », Impact of Science on Society, 160, 1990, p. 357-367.

4. Jacques Revel, «Histoire et sciences », Annales. Économies, sociétés, civilisations, $30^{\mathrm{e}}$ année, sept.-oct. 1975, av.-pr., p. 934. 
Jacques Le Goff et Pierre Nora, figurait également en bonne position un chapitre sur les sciences dont la rédaction avait été confiée à Michel Serres, destiné à succéder à Suzanne Bachelard à la chaire d'histoire des sciences de la Sorbonne ${ }^{5}$. Boulevard Raspail, François Furet avait déployé un effort de renouveau en invitant Kuhn lui-même et nombre d'historiens des sciences américains à donner des séminaires à Paris.

Une opération de réflexion de longue haleine fut même lancée sous une forme interdisciplinaire. C'était un séminaire à mi-chemin entre cognitivisme et herméneutique qu'animait l'archéologue Jean-Claude Gardin et auquel avaient été conviés des sociologues, des psychologues, des philosophes ainsi que des historiens des sciences : de Gérard Lemaine à Pierre Jacob, de Claire Salomon-Bayet à Serge Moscovici et à Claude Petitot, ainsi qu'Ernest Coumet. L'initiative n'eut pas le succès qu'on avait espéré et fut vite abandonnée.

Si je rappelle ces essais réitérés de faire démarrer un débat de fond entre l'histoire, la philosophie et les sciences, c'est pour situer la manière dont Coumet tenta, selon moi, de réorienter le problème dès le départ de son enseignement.

La parution, en 1977, du recueil d'essais de Kuhn, La Tension essentielle, lui fournit la possibilité de reprendre les fils qui reliaient la théorie de la structure des révolutions scientifiques avec les concepts élaborés par les historiens des sciences de la génération précédente, notamment ceux d'Alexandre Koyré et de Jean Piaget, au sujet de la notion de la nature de la science moderne. Il s'avérait que Kuhn n'avait pas déclaré toutes ses dettes intellectuelles à l'égard de ses prédécesseurs. Les identifier permettait de relativiser une épistémologie historique comme la sienne, alors tenue pour parole d'évangile, en la mettant en perspective avec les questions et les modèles qui avaient permis de penser d'une manière critique la science moderne comme une histoire de mutations et de ruptures.

À partir de là, une grande partie des séminaires de Coumet aura été consacrée à reconstituer de larges pans de l'histoire des sciences: d'Auguste Comte à Pierre Duhem, de Robert K. Merton à Frances Yates, de Pierre Lenoble à Joseph Needham. De ces recherches témoignent aussi bien son étude sur «Paul Tannery et l'organisation de l'histoire des sciences en France» que celle sur «Alexandre Koyré. La Révolution scientifique introuvable? $»^{6}$.

5. Michel Serres, «Les Sciences », in Jacques Le Goff et Pierre Nora, dir., Faire de l'histoire. T. II : Nouvelles approches, Paris, Gallimard, 1974, p. 203-228.

6. Voir respectivement : Revue de synthèse, III ${ }^{\mathrm{e}}$ sér., t. CII, 101-102, janv.-juin 1981, p. 87123 ; et «Science. The renaissance of a history. Proceedings of the international conference Alexandre Koyré, Paris, Collège de France, 10-14 june 1986 », éd. Pietro Redond, nº spéc. History and Technology, vol. IV, 1-4, 1987, p. 497-529. 
Ce dernier travail a clairement renouvelé l'interprétation traditionnelle de l'auteur des Études galiléennes, et je voudrais lui rendre hommage en disant l'effet qu'il a produit sur moi, qui considérais comme beaucoup d'autres que l'apport essentiel de Koyré comme historien des sciences résidait dans son approche d'histoire anthropologique par une analyse conceptuelle fine des textes et par l'étude des conditions culturelles de leur élaboration — « les substructures de la pensée scientifique » comme les appelait Koyré. On faisait remonter cette empathie singulière avec la science du passé à la formation phénoménologique de l'auteur et notamment à son souci pour «l'unité de la pensée » de l'âge classique, échappant à une démarcation anachronique entre histoire des pensées religieuses, philosophiques et scientifiques.

Coumet s'attaquait par contre à ce noyau dur de l'œuvre de Koyré en histoire des sciences, à savoir sa catégorie de «révolution scientifique moderne », qu'il caractérisait a priori et polémiquement dès le début des Études galiléennes en termes d'infinitisation et de géométrisation du monde.

Au fil de ses livres, Koyré n'a fait que guetter et talonner, de Copernic à Galilée et de Descartes à Newton, ce processus révolutionnaire dont il ne faisait que constater l'inachèvement et l'avènement toujours remis à l'avenir. D'après la reconstruction que proposait Coumet de cette démarche, un tel effort prescriptif pour interpréter la science moderne comme une révolution décalée d'un savant à l'autre s'inspirait de la critique de la formation des concepts historiques par laquelle Max Weber cherchait pour chaque époque à construire un modèle, voire un tableau ou un idéaltype, afin de caractériser le processus de rationalisation du monde moderne.

En l'occurrence, la révolution scientifique dont Koyré cherchait l'avènement idéal à rebours était un modèle de science de type einsteinien. L'abstraction de la géométrie de l'espace-temps de la relativité était la révolution intellectuelle dont Koyré poursuivait de près les prophéties. Autrement dit, l'histoire des sciences de Koyré était de style bien plus récurrent que ce que l'on avait soupçonné ${ }^{7}$.

Visiblement, cette remise en question de la révolution de la science moderne allait dans la même direction que la critique de la notion de Révo-

7. Sur Alexandre Koyré, voir Alexandre Koyré. L'avventura intellettuale, a cura di Carlo VINTI, Naples, Edizioni scientifiche italiane, 1994, et les documents et analyses publiés par Paola Zambelli, « Koyré, Hannah Arendt e Jaspers », Nouvelles de la république des lettres, 1, 1997, p. 131-156; ID., « Alexandre Koyré alla scuola di Husserl a Gottinga », Giornale Critico della Filosofia Italiana, 77, 1999, p. 303-354; ID., " Alexandre Koyré im "Mekka der Mathematik" », NTM, vol. VII, 1999, p. 208-230; ID., «Introduction», in Jean-François StOFFel, Bibliographie d'Alexandre Koyré, Florence, Olschki, 2000, p. vII-Xx. Contrairement au chantier de recherche désormais ouvert sur Koyré, il faut constater encore l'absence relative d'apports documentaires et de discussion sur Gaston Bachelard. 
lution française menée par Furet ${ }^{8}$. Visiblement aussi, ces deux démarches de réflexion sur l'épistémologie historique n'auront été que parallèles. Et il est à regretter que dans le milieu des historiens n'ait pas été suffisamment reconnu l'objectif que Coumet s'était donné, c'est-à-dire d'examiner l'entrelacement de l'histoire, de la philosophie et de la sociologie historique à l'œuvre dans le fondement des théories de l'histoire des sciences ${ }^{9}$.

$\mathrm{Si}$ je peux me permettre de terminer par un souhait, ce serait qu'au moins partiellement, puissent faire aussi l'objet de publication d'autres travaux d'histoire de l'histoire des sciences abordés par Ernest Coumet au fil des séminaires qu'il consacra à la Révolution scientifique et au tournant intellectuel de 1900. Ceux qui ont assisté à ces séminaires en gardent le souvenir d'une expérience intellectuelle privilégiée aux côtés d'un maitre d'une grande générosité intellectuelle. J'espère qu'ils ne seront pas les seuls à pouvoir en bénéficier.

Pietro REDONDI, Dipartimento di Filosofia, Università degli Studi di Bologna, Via Zamboni, 38, I-40126 Bologne (décembre 2000).

8. François Furet, Penser la Révolution française, Paris, Gallimard, 1978.

9. Sur les nœuds entre histoire des sciences et histoire, voir la contribution de Roger CHARTIER, « Histoire intellectuelle et histoire des mentalités. Trajectoires et questions », à la journée organisée par Jacques Roger, «Histoire des sciences et mentalités », Revue de synthèse, III $^{\mathrm{e}}$ sér., t. CIV, 111-112, juil.-déc. 1983, p. 277-307. Plus récemment, voir les articles de Catherine Chevalley, Pierre Jacob, Gérard Jorland et Dominique Pestre dans «Comment écrire l'histoire des sciences? », Le Débat, 102, 1998, p. 53-106. 